Article

\title{
Casein-Conjugated Gold Nanoparticles for Amperometric Detection of Leishmania infantum
}

\author{
Mohamed Fethi Diouani ${ }^{1,3}$, Oussama Ouerghi ${ }^{2,3, * \mathbb{C}}$, Kamel Belgacem ${ }^{1}$, Maher Sayhi ${ }^{1,3}$ (D), \\ Radu Ionescu ${ }^{4}$ and Dhafer Laouini ${ }^{3,5}$ \\ 1 Institut Pasteur de Tunis, LR11IPT03, Laboratory of Epidemiology and Veterinary Microbiology (LEMV), \\ Tunis-Belvédère 1002, Tunisia; fethi.diouani@pasteur.tn (M.F.D.); kamelbelgacem85@gmail.com (K.B.); \\ sayhimaher@ymail.com (M.S.) \\ 2 Prince Sattam Bin Abdulaziz University, Al Kahrj 11942, Saudi Arabia \\ 3 Université Tunis El Manar, Tunis 1068, Tunisia; dhafer.laouini@pasteur.tn \\ 4 The Ångström Laboratory, Division of Solid State Physics, Department of Engineering Sciences, \\ Uppsala University, 75121 Uppsala, Sweden; radu.ionescu@angstrom.uu.se \\ 5 Institut Pasteur de Tunis, LR11IPT02, Laboratory of Transmission, \\ Control and Immunobiology of Infections (LTCII), Tunis-Belvédère 1002, Tunisia \\ * Correspondence: o.ouerghi@psau.edu.sa or oussamaouerghi@yahoo.com
}

Received: 17 March 2019; Accepted: 25 April 2019; Published: 27 May 2019

check for updates

\begin{abstract}
Sensitive and reliable approaches targeting the detection of Leishmania are critical for effective early diagnosis and treatment of leishmaniasis. In this frame, this paper describes a rapid quantification assay to detect Leishmania parasites based on the combination of the electrocatalytic ability of gold nanoparticles (AuNPs) to act as a catalyst for the hydrogen formation reaction along with the specificity of the interaction between casein and the major surface protease of the Leishmania parasite, GP63. First, pure and casein-modified AuNPs were prepared and characterized by scanning electron microscopy and ultraviolet-visible spectroscopy. Then, casein-conjugated AuNPs were incubated with Leishsmania parasites in solution; the formed complex was collected by centrifugation, treated by acidic solution, and the pelleted AuNPs were placed on screen-printed carbon electrodes (SPCEs) and chronoamperometric measurements were carried out. Our results suggest that it is possible to detect Leishmania parasites, with a limit less than 1 parasite/mL. A linear response over a wide concentration interval, ranging from $2 \times 10^{-2}$ to $2 \times 10^{5}$ parasites $/ \mathrm{mL}$, was achieved. Additionally, a pretreatment of Leishmania parasites with Amphotericin B, diminished their interaction with casein. This findings and methodology are very useful for drug efficacy assessment.
\end{abstract}

Keywords: Leishmania infantum; gold nanoparticles; casein; chronoamperometry; GP63

\section{Introduction}

Leishmania (L.) is an intracellular protozoan parasite transmitted by phlebotomine sandflies. It causes a wide range of human diseases, ranging from cutaneous lesions to visceral dissemination. As reported by the World Health Organization (WHO), leishmaniasis is one of the six most important diseases worldwide, putting at risk over 350 million people and causing 1.6 million new cases annually in 98 countries, including developed and developing countries alike [1,2]. However, the majority of cases (more than 90\%) occur in low-income countries suffering from the lack of necessary health resources for effective diagnosis and control of the disease [2]. In the Mediterranean region, including Tunisia, visceral leishmaniasis (VL) is mainly due to L. infantum species, with dogs as the major natural reservoir of the parasite and playing a crucial role in the transmission and spread of the disease [3]. Conventional golden standard assays for leishmaniasis diagnosis include historically the microscopic 
approach, based on the observation or isolation of the infectious agent for in-lab confirmation of the infection; serology-based assays, based on the detection of specific antibodies against leishmaniosis infection; and molecular-based approaches, based on nucleic acids and often using polymerase chain reaction (PCR) techniques [4-7]. Despite their sensitivity, these techniques require specific and costly equipment, highly-skilled staff, and long turnaround times. Due to the limitations of these conventional detection methods, it is necessary to develop reliable diagnostic assays. The advantages acquired from nanotechnology and its application for the development of biosensors can be used friendly without any needs for qualified technicians. These devices could significantly support the growing efforts devoted to decrease the impact of the disease on public health for humans and animals alike.

Basically, the life cycle of Leishmania involves two phases. In the first phase, motile and flagellated promastigotes reside in the midgut of phlebotomine sandflies. Promastigotes are then injected into a mammalian host by the vector (phlebotomine sandflies) during an accidental feeding. During the second phase, promastigotes are internalized in the macrophages of the mammalian host, where they transform into nonmotile amastigotes [8].

Leishmania species express an abundant surface glycoprotein, known as leishmanolysin and referred to as GP63 [9]. The latter is assumed to be a ligand involved in the interactions between the parasite and the host's immune system, including components of the complement system and the macrophage surface [10]. Thus, GP63 weakens the activity of macrophages tolerating intracellular invasion, allowing the spread and the survival of the parasite. GP63/leishmanolysin, also known as the major surface protease (MSP), is a zinc-dependent metalloprotease overexpressed at the surface of the parasite, or directly secreted by Leishmania. This enzyme is expressed predominantly in the promastigote stage, whereas it drops to low levels as the parasite transmutes to its amastigote form [11]. Another important aspect of GP63 is its ability to degrade various substrates, such as casein, hemoglobin, gelatin, fibrinogen, and albumin [12]. A previous study reported casein as the best substrate for detecting GP63-like proteins in Herpetomonas megaseliae parasites [13].

Caseins, the most abundant proteins in milk, are amphiphilic block copolymers futured by a large number of hydrophobic or hydrophilic amino acid residues. Thanks to their amphiphilicity in aqueous solution, they experience a high ability to self-assemble into stable micelles $[14,15]$. Stability against aggregation of casein micelles, mainly controlled by the steric repulsion between the polypeptide brushes, makes them useful for the production of stabilized gold nanoparticles (AuNPs) in aqueous solutions [16]. However, in addition to offering high stability for the system, the conjugation of caseins with AuNPs also provides these nanoparticles with the biocompatible functionalities that are often required for many biological applications $[17,18]$. Previous work reported a casein-based AuNP assay to assess the E. coli-casein interaction by monitoring catalytic current associated with the hydrogen formation reaction performed on screen-printed carbon electrodes [19].

Among the wide variety of nanoparticles, AuNPs have been the focus of many research studies due to their exceptional physicochemical properties, i.e., structural, electric, optic, magnetic, and catalytic, which make them very attractive for versatile bioassays and biosensors applications [20-23]. Particularly, the catalytic activity of AuNPs for the hydrogen reaction formation has paved the way for the building of highly sensitive sensors with large sensing properties [24-28].

Conceptually, the AuNPs, deposited on the surface of a screen-printed carbon electrode, release a large number of $\mathrm{Au}^{3+}$ ions from each gold nanoparticle. These ions provide free electroactive sites to the protons $\left(\mathrm{H}^{+}\right)$present in the acidic medium $(\mathrm{HCl})$. The protons were then catalytically reduced to hydrogen under an adequate potential, producing a shift in the cathodic current. For biodetection applications, the target biomolecule reacts with its specific probes that were already labeled by gold nanoparticles. The formed complex, when treated by an acid, releases gold nanoparticle that can be quantified by chronoamperometry, which in turn allows quantification of target biomolecules. In other words, target biomolecules are indirectly quantified via the quantification of the gold nanoparticles anchored to the probes. 
Encouraged by the above-mentioned studies, the present work is based on the dual use of AuNPs as nanocarriers and as electrocatalytic labels with the potentiality of casein to act as a substrate for the GP63 protease. We used these advantages to design a sensing assay for Leishmania parasites detection. In fact, casein-conjugated AuNPs were incubated with Leishmania parasites at different concentrations. The formed complexes were collected by centrifugation, treated by acidic solution then the collected AuNPs were placed on the working electrode surface of screen-printed carbon electrodes (SPCEs) and chronoamperometric measurements were carried out. Principally, we processed by monitoring the cathodic current associated with the reduction of protons $\left(\mathrm{H}^{+}\right)$to hydrogen, in acidic medium, amplified by the catalytic effect of AuNPs when a suitable potential is imposed to the working electrode. The recorded current is correlated with the concentration of AuNPs quantified inside the test medium, and in turn quantifies Leishmania parasites. Such a method promises substantial potential for use in human and veterinary medicine applications, particularly in limited-resources settings.

\section{Materials and Methods}

\subsection{Reagents and Apparatuses}

Caseino-glycopeptide (CGP), hydrogen tetrachloroaurate (III) trihydrate $\left(\mathrm{HAuCl}_{4}{ }_{3} \mathrm{H}_{2} \mathrm{O}\right.$, $99.9 \%$, trisodium citrate $\left(\mathrm{Na}_{3} \mathrm{C}_{6} \mathrm{H}_{5} \mathrm{O}_{7} .2 \mathrm{H}_{2} \mathrm{O}\right)$, Amphotericin $\mathrm{B}(\mathrm{AmB}), \mathrm{RPMI}-1640$ medium, gentamicin, and fetal bovine serum were purchased from Sigma-Aldrich (Saint-Quentin, France). Potassium dihydrogen phosphate $\left(\mathrm{KH}_{2} \mathrm{PO}_{4}\right)$ and dipotassium hydrogen phosphate $\left(\mathrm{K}_{2} \mathrm{HPO}_{4}\right)$ reagents were purchased from Fluka (Saint-Quentin, France) and were used to prepare phosphate buffer solutions (0.01 M PBS, $\mathrm{pH} 6.8$ and $\mathrm{pH}$ 7.4).

The L. infantum parasites (IPT1 strain) were routinely maintained in culture in our laboratory and were collected from our previous epidemiological investigation on canine leishmaniasis in endemic area of Tunisia [29].

Cyclic voltammetry and chronoamerometry experiments were performed on a computerized PGZ 301 Voltalab 40 potentiostat purchased from radiometer analytical instrument S.A. (Hach Lange France, Marnes-La-Vallée, France) Screen-printed carbon electrodes (SPCEs, DRP-110), as well as their specific connector to the potensiostat, were acquired from DropSens (LIanera, Asturias, Spain). The SPCEs consist of a three-electrode test cell, printed on ceramic substrates $(33 \times 10 \times 0.5 \mathrm{~mm})$ including a circular-shaped working electrode with a diameter of $4 \mathrm{~mm}$, a counter and a pseudo reference electrode made of silver. Working and counter electrodes are both made of carbon ink. A ring-shaped insulating layer around the working electrode with a capacity of $50 \mu \mathrm{L}$ was incorporated into the SPCES to form the reservoir of the test cell.

Optical characterization of the as-prepared AuNPs was performed by a UV-Vis spectrophotometer (Single Beam LI-295, Lasany, India), while morphological characterization was carried out by a transmission electron microscope (TEM) JEM-1011 from Jeol Ltd., (Tokyo, Japan) operating under high vacuum conditions and an accelerating voltage of $150 \mathrm{kV}$.

X-ray photoelectron spectroscopy (XPS) analysis of casein-conjugated AuNPs was carried out using a K-Alpha XPS system (Thermo Fisher Scientific, Waltham, MA, USA) that is equipped with a microfocused monochromatic Al Ka X-ray source (1486.6 eV).

\subsection{Leishmania Parasite Culture}

L. infantum promastigotes were cultured in RPMI-1640 medium, purchased from Sigma-Aldrich, supplemented with $10 \%$ heat-inactivated fetal bovine serum (FBS) and $1 \%$ gentamicin $(50 \mathrm{mg} / \mathrm{mL})$ at $23^{\circ} \mathrm{C}$. Cultures were seeded by inoculation with early stationary phase promastigotes at starting density of $1 \times 10^{6}$ parasites $/ \mathrm{mL}$. Neubauer brightline hemocytometer was used to count the parasites daily. 


\subsection{AuNPs Preparation and Functionalization}

AuNPs were prepared as described in our previous work [30] via the reduction reaction of a tetrachloroauric acid with trisodium citrate, following a procedure initially reported by Turkevich with few modifications [31]. In brief, a volume of $200 \mathrm{~mL}$ from a $0.01 \% w / v \mathrm{HAuCl}_{4}$ solution was heated until boiling while stirring continuously. Then, $5 \mathrm{~mL}$ of a $1 \% w / v$ trisodium citrate solution was supplemented rapidly to the latter prepared solution. During this process, the color of the solution changes from yellow to deep red. Finally, the resultant solution was left cooling with stirring, then stored in dark.

The casein-AuNPs conjugates were prepared as previously described by Espinoza-Castañeda [19], who described the optimum conditions to avoid agglomeration caused by casein micelles and the encapsulation of the AuNPs by micelles. Typically, a casein solution of a concentration of $0.1 \mathrm{mg} / \mathrm{mL}$ together with a casein/AuNPs concentration ratio of 3:1 are appropriate. The incubation of casein with AuNPs solutions was accomplished by gentle stirring, for $30 \mathrm{~min}$ at room temperature. Casein excess was discarded by centrifugation of the former solution at $14000 \mathrm{rpm}$. The collected pellet was diluted in $0.01 \mathrm{M}$ PBS, at $\mathrm{pH} 6.8$, and the obtained solution was stored in dark at $4{ }^{\circ} \mathrm{C}$. Spectrophotometric analyses were performed by loading $1 \mathrm{~mL}$ suspension of the AuNPs and the casein@AuNPs conjugates, separately in Quartz cells and scanning the wavelength from $450 \mathrm{~nm}$ to $650 \mathrm{~nm}$.

\subsection{Incubation of the Parasites with casein@AuNPs and Leishmania Quantification}

Quantitative analyses of Leishmania parasites were performed through a chronoamperometric approach and followed a previously optimized procedure [19]. Such analytical procedure is schematically depicted in Figure 1 . Briefly, $500 \mu \mathrm{L}$ casein-AuNPs were incubated with $500 \mu \mathrm{L}$ of different concentrations of Leishmania (from $2 \times 10^{-2}$ to $2 \times 10^{6}$ parasites $/ \mathrm{mL}$ ) for $30 \mathrm{~min}$ at room temperature under gentle stirring. At this stage, the AuNPs are attached to Leishmania parasites via casein-GP63 interactions. The obtained solution was then centrifuged at $3000 \mathrm{rpm}$ to eliminate the unbounded AuNPs. After removing the supernatant, the collected pellet, containing the AuNPs-casein-Leishmania complex, was reinjected in $100 \mu \mathrm{L}$ of $0.01 \mathrm{M} \mathrm{PBS}$ at $\mathrm{pH}$ 7.4. Chronoamperograms were achieved by putting down a mixture containing $25 \mu \mathrm{L}$ of the casein-AuNPs-Leishmania complex with $25 \mu \mathrm{L}$ of $2 \mathrm{M} \mathrm{HCl}$ onto the working electrode. Oxidative pretreatment of AuNPs was initially accomplished by application of potential of $+1.35 \mathrm{~V}$ for $60 \mathrm{~s}$. Next, a potential of $-1 \mathrm{~V}$ was applied to the working electrode for $100 \mathrm{sec}$ and the generated current was recorded. Subsequently, the hydrogen formation reaction was investigated through the change in the current arising during the catalytic process using a chronoamperometric approach. 


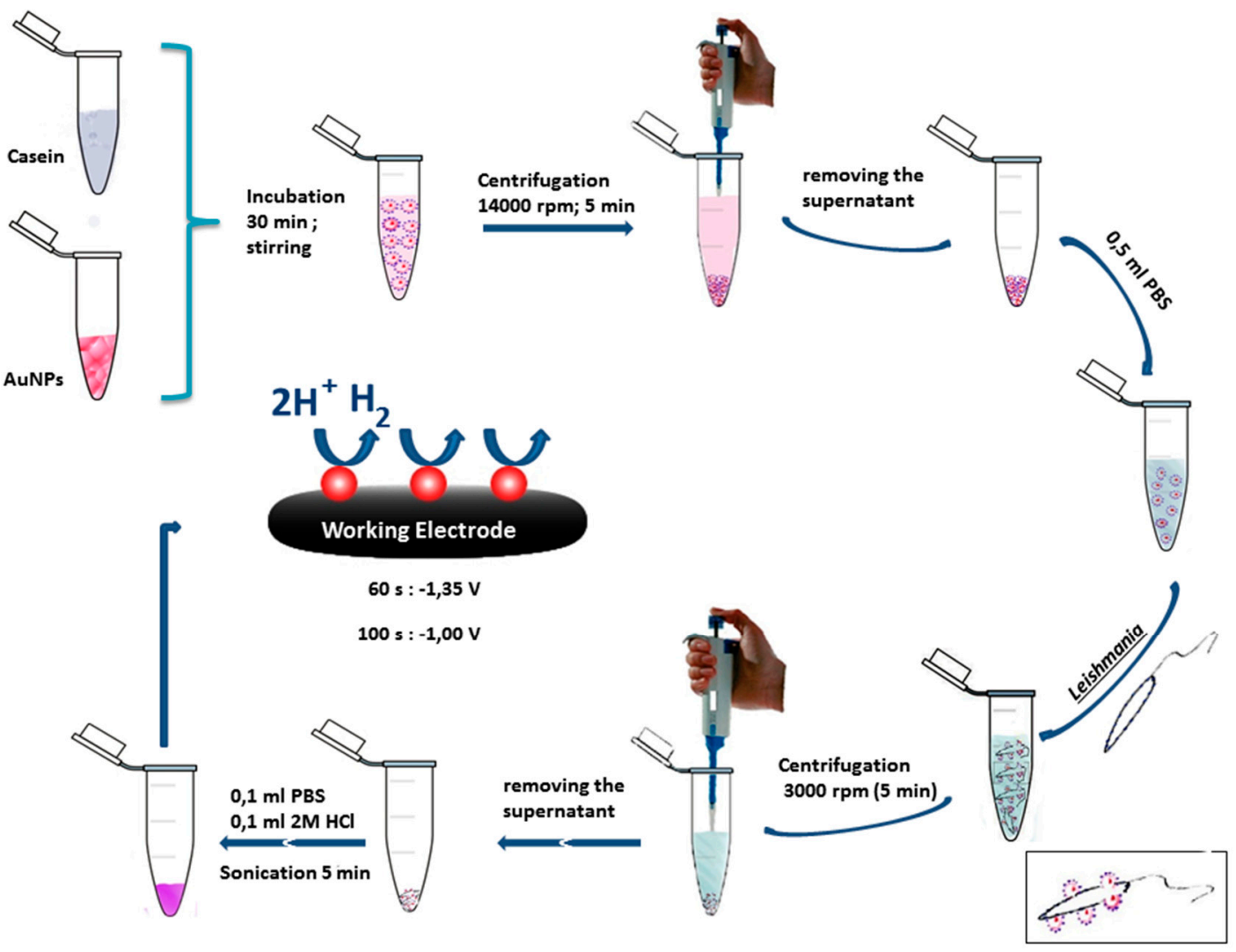

Figure 1. Schematic illustration of the chronoamperometric detection principle of Leishmania infantum parasites through hydrogen evolution reaction catalyzed by gold nanoparticles.

\subsection{Electrochemical Assessment of the Effect of Amphotericin B Pretreatment on Leishmania-casein Interaction}

To evaluate the effect of Amphotericin B (AmB) on casein-Leishmania interaction, chronoamperometric responses were recorded at different incubation times of the parasite using a fixed concentration of the antibiotic. Leishmania parasites $\left(10^{4} / \mathrm{mL}\right)$ were mixed with $0.3 \mu \mathrm{g} / \mathrm{mL}$ of $\mathrm{AmB}$ for $10,30,120,240$, and $360 \mathrm{~min}$ at $37^{\circ} \mathrm{C}$. Then, casein-AuNPs were added to the mixture for $30 \mathrm{~min}$ at room temperature with gentle stirring. Pellets from the reaction mixtures were then collected by centrifugation at $3000 \mathrm{rpm}$ for $10 \mathrm{~min}$, and resuspended in PBS/HCl. Finally, chronoamperometric responses were recorded as described above.

\section{Results and Discussion}

\subsection{Characterization of Pure and casein-Capped AuNPs}

Owing to their extraordinary electrocatalytic activity, AuNPs have been widely exploited as labels for various biorecognition and biosensing processes [32,33]. However, their catalytic performance was found to depend significantly on the particle size [30]. Moreover, the grain size and morphology of the nanoparticles were controlled by the synthesis method conditions [34]. Besides, casein molecules were reported to bind to AuNPs surface, which subsequently led to the formation of casein-AuNP conjugates [16]. Herein, the synthetized AuNPs as well as the casein-AuNPs conjugates were characterized using TEM, UV-Vis and XPS.

As shown in Figure 2a, the TEM image of the bare AuNPs shows that these colloidal nanoparticles are well dispersed and roughly spherical having an average diameter of $\sim 14 \mathrm{~nm}$ determined from the size distribution histogram corresponding to the analysis of the above-mentioned image (Figure 2b). Furthermore, as the electrocatalytic activity of AuNPs is highly affected by their size, sufficiently narrowed size distribution is required to achieve reproducible catalytic effects. For this 
reason, each experiment in this study was conducted using AuNPs extracted from the same batch to reduce the unavoidable dispersion in the standard deviation of the obtained results.
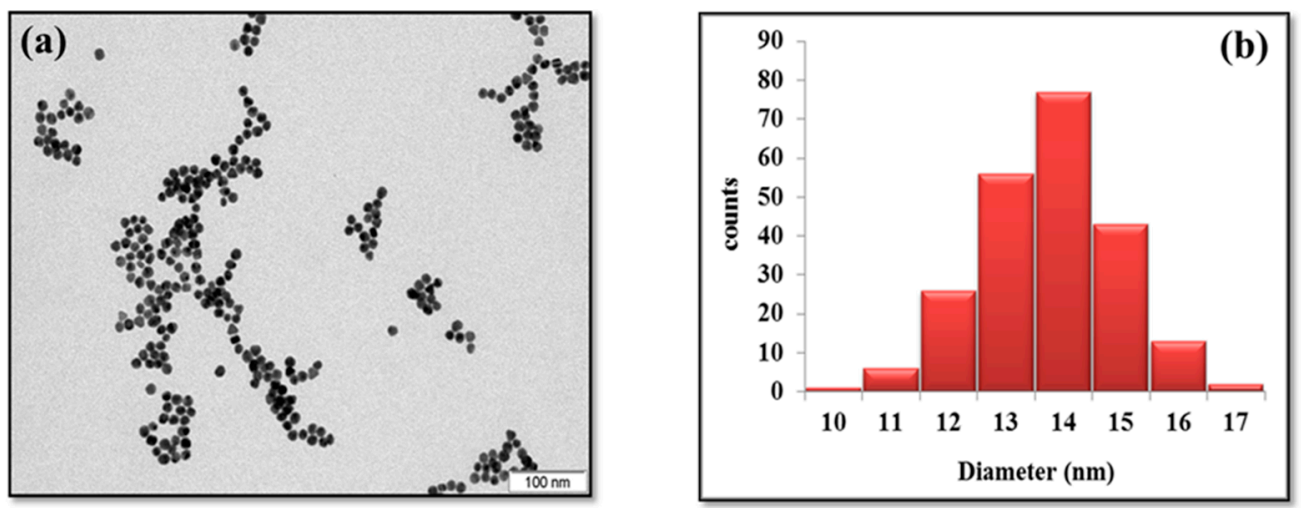

Figure 2. (a) TEM image of the prepared AuNPs; (b) AuNPs size histogram distribution.

Besides, the UV-visible absorption spectrum revealed that the maximum absorption shifted from $521 \mathrm{~nm}$ for the pure AuNPs to $527 \mathrm{~nm}$ after their conjugation with casein (Figure 3a). Such an observed shift to longer wavelengths along with the broadening of the surface-plasmon resonance (SPR) peak could be ascribed to the alterations of the local dielectric constant around the AuNPs because of their conjugation with casein. Moreover, TEM image of the casein-AuNPs conjugates revealed that their size is slightly higher $(18 \pm 2 \mathrm{~nm})$ with respect to the size of the unmodified AuNPs. This result is consistent with the observed UV-Vis shift and suggests the efficient coating of the AuNPs by casein.
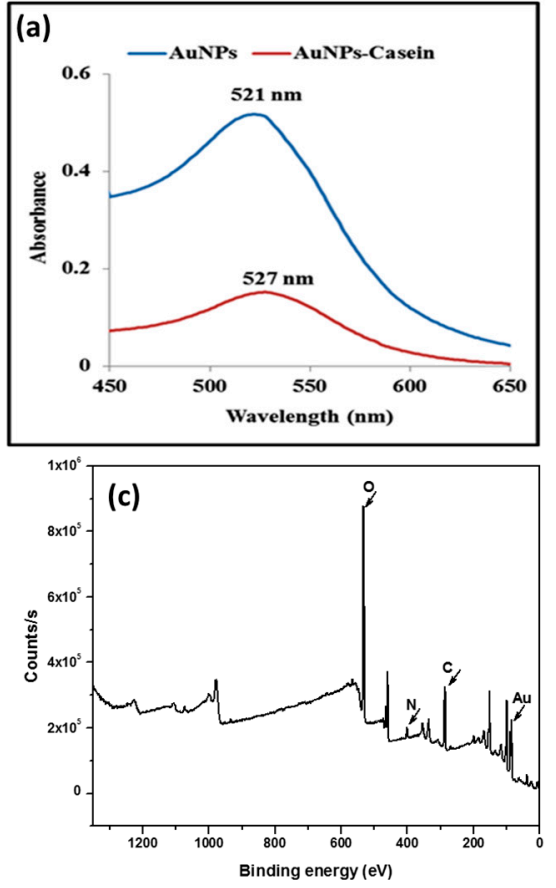
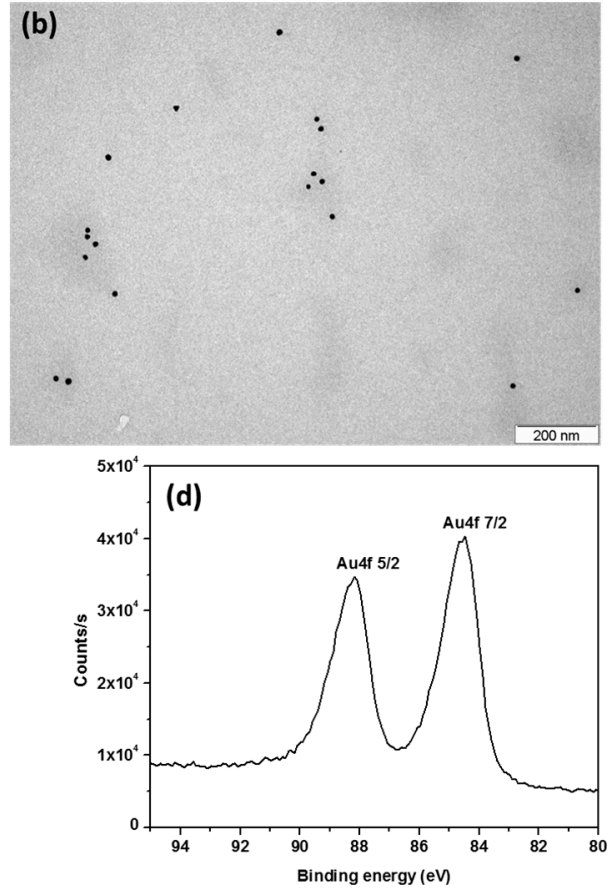

Figure 3. (a) UV-visible spectra of bare AuNPs and the casein-AuNPs conjugates; (b) TEM image of the casein-AuNPs; (c) The whole XPS spectrum of casein@AuNPs; (d) XPS spectrum showing the two peaks of the Au4f spin-orbit doublet binding energy.

Surface chemical compositions of the casein-AuNPS conjugates were further evaluated by X-ray photoelectron spectroscopy (XPS). The XPS spectrum of casein-AuNPs deposited on a silicon oxide substrate was shown in Figure 3c. Results reveal the presence of gold, carbon, nitrogen and oxygen, 
indicating that the AuNPs were successfully capped by casein, which is in agreement with the UV-Vis and the TEM results.

Furthermore, Figure $3 d$ shows the XPS spectra of the Autf doublet $\left(4 f_{7 / 2}\right.$ and $\left.4 f_{5 / 2}\right)$ for AuNPs capped with casein. The $A u 4 f_{7 / 2}$ and $A u 4 f_{5 / 2}$ peaks appeared at 84.6 and $88.3 \mathrm{eV}$, respectively, which are the typically characteristic of the pure metallic $\mathrm{Au}^{0}[35,36]$, confirming the formation of AuNPs.

\subsection{Electrocatalytic Activity of AuNPs Towards Hydrogen Ions Reduction}

Even though gold is an inert metal, the high surface area to volume ratio, surface properties $[37,38]$, and nanoscale dimensions [39] enhance considerably the catalytic activity of the derivative AuNPs, resulting, in turn, to amplified electrical signals [40,41]. The electrocatalytic activity of AuNPs towards the proton reduction was initially investigated by cyclic voltammetry. Indeed, $25 \mu \mathrm{L}$ of a $2 \mathrm{M} \mathrm{HCl}$ was added to $25 \mu \mathrm{L}$ AuNPs solutions of various concentrations. The derivative mixture was deposited on the working electrode of the SPCEs electrodes. Cyclic voltammetry measurements were then performed by scanning the potential from +1.35 to $-1.40 \mathrm{~V}$ at a scan rate of $50 \mathrm{mV} / \mathrm{s}$. Likewise, the background signal (blank experiment) was carried out by deposing $50 \mu \mathrm{L}$ of a $1 \mathrm{M} \mathrm{HCl}$ onto the working electrode of the SPCEs.

Data of Figure 4a show that the hydrogen reduction reaction, in the test medium, arises from a potential around $-1 \mathrm{~V}$ as displayed in the background CV. However, when AuNPs are placed onto the working electrode, the reduction potential for hydrogen ion shifted to less negative potentials (more than $300 \mathrm{mV}$ ) as the concentration of AuNPs increases. Likewise, as shown in Figure 4b, because of the catalytic activity of the AuNPs, higher current is observed with increasing AuNP concentration.
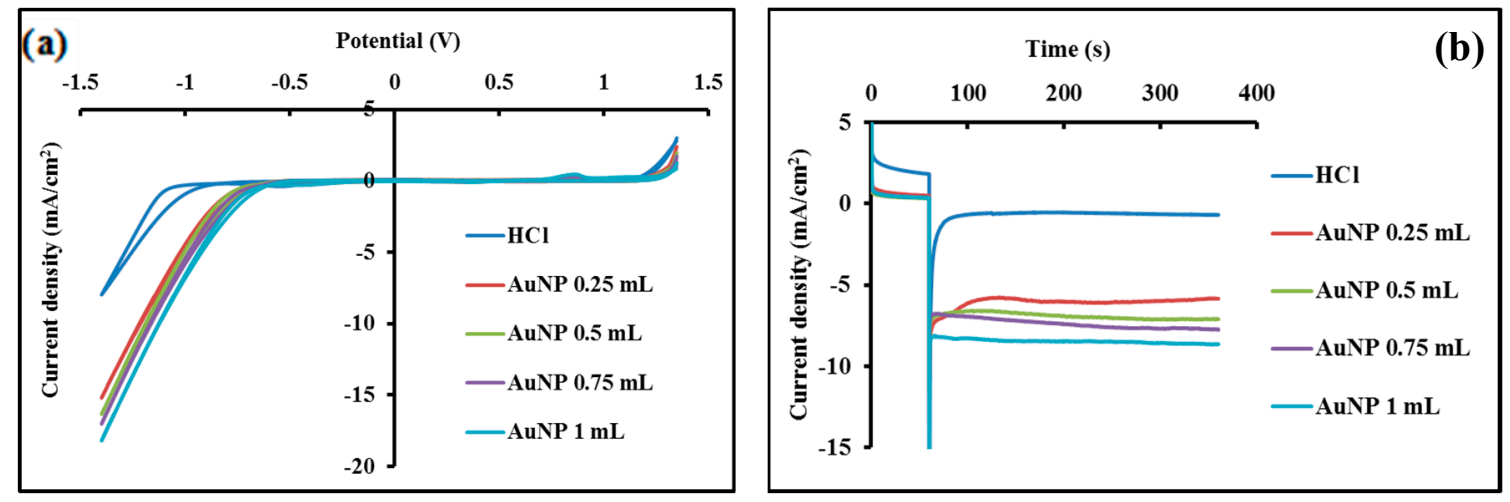

Figure 4. (a) Cyclic voltammograms recorded from +1.35 to $-1.40 \mathrm{~V}$ at a scan rate of $50 \mathrm{mV} / \mathrm{s}$ for a $1 \mathrm{M} \mathrm{HCl}$ solution and for increasing concentrations of AuNPs in $1 \mathrm{M} \mathrm{HCl}$. (b) Chronoamperograms recorded by applying a potential of $-1.00 \mathrm{~V}$ for $300 \mathrm{~s}$, using a $1 \mathrm{M} \mathrm{HCl}$ solution and the same AuNPs concentrations in $1 \mathrm{M} \mathrm{HCl}$.

Additional investigations concerning the catalytic activity of AuNPs were performed using chronoamperometry approach. The recorded chronoamperograms were accomplished for the as-prepared mixture, and achieved by addition of $25 \mu \mathrm{L}$ of $2 \mathrm{M} \mathrm{HCl}$ to $25 \mu \mathrm{L}$ AuNP solutions of various concentrations, while imposing a constant potential of $+1.35 \mathrm{~V}$ on the working electrodes for $1 \mathrm{~min}$. This pretreatment step is crucial for obtaining the greatest catalytic activity and can be explained by the fact that, whenever an oxidative potential is imposed to the working electrode, some atoms from the surface of AuNPs are dissolved in the solution and $\mathrm{Au}^{3+}$ ions are formed. These released ions in the solution could enhance the catalytic effect on the hydrogen formation reaction [41]. The cathodic current generated was registered during $300 \mathrm{~s}$, while a constant potential of $-1 \mathrm{~V}$ was applied to the working electrode. The background signal was performed through the measurement of the cathodic current produced by a $50-\mu \mathrm{L}$ solution of $\mathrm{HCl}(1 \mathrm{M})$ placed onto the working electrode surface, while maintaining the same procedure as described above. 
The generated current throughout the hydrogen ion reduction process appears to be closely associated with the concentration of AuNPs in the test solution placed onto the working electrode. The amplitude of the recorded current (absolute value), taken at $100 \mathrm{~s}$, was undertaken as the assay's analytical signal.

\subsection{Detection of Leishmania Parasites}

Usually, the immobilization of immune reagents, such as antibody or antigen on an electrode surface for target analyte detection, is highly required in immunosensor technology. Advantageously, in the present work, labeling of Leishmania parasites was achieved in suspension, which allows casein-AuNPs conjugates to interact readily and rapidly with GP63 proteins overexpressed at the surface of the parasite. GP63 proteins were previously used as a recognition element in the design of sensing assays for Leishmania parasites [42,43].

Furthermore, it is worth mentioning that the casein-AuNPs-Leishmania complex can be destroyed by the acidic process achieved prior to the last detection step. Actually, acidic treatment performed after any biological reaction is a commonly useful approach for the detection of nanoparticles [44]. Biological systems subject to this process are denatured, whereas nanoparticles remain in the detection solution, retain their catalytic ability and their concentration reveals the amount of the target analyte. Following the incubation protocol, Leishmania parasites were detected by measurement the chronoamperometric current arising from the catalytic process associated with the hydrogen evolution reaction performed in $1 \mathrm{M} \mathrm{HCl}$ solution and catalyzed by AuNPs labels.

Figure 5 shows the evolution of the analytical signal with the concentration of Leishmania parasites, over the interval $2 \times 10^{-2}$ and $2 \times 10^{6}$ parasites $/ \mathrm{mL}$. A linear relationship was observed between $2 \times 10^{-2}$ and $2 \times 10^{5}$ parasites/mL with a limit of detection (LOD) of about 0.55 parasite/mL and a correlation coefficient of 0.97 . LOD was calculated using the following equation, LOD $=3 \sigma / \mathrm{s}$, where $\sigma$ is the standard deviation of the blank measurement and $\mathrm{s}$ is the slope of the linear part of the calibration curve. The reproducibility of the method shows a relative standard deviation (RSD) around $4 \%$, obtained for 3 repetitive assays. The low limit of detection (less than 1 parasite/mL) is likely due to the fact that GP63 proteins can also be secreted outside by Leishmania parasites in the form of microvesicles [45]. Indeed, microvesicle-based secretion appears to be a general mechanism for the secretion of proteins by protozoan parasites [46,47]. It has been previously reported that proteins enclosed inside these microvesicles can be involved in the recognition of parasite infection and pathogen survival is several diseases including malaria and chagas disease. Consequently they could be used for disease treatment $[48,49]$. On the other hand, these extracellular vesicles are highly immunogenic, and could hence be used as appropriate biomarkers for early detection of parasitic diseases [46]. 


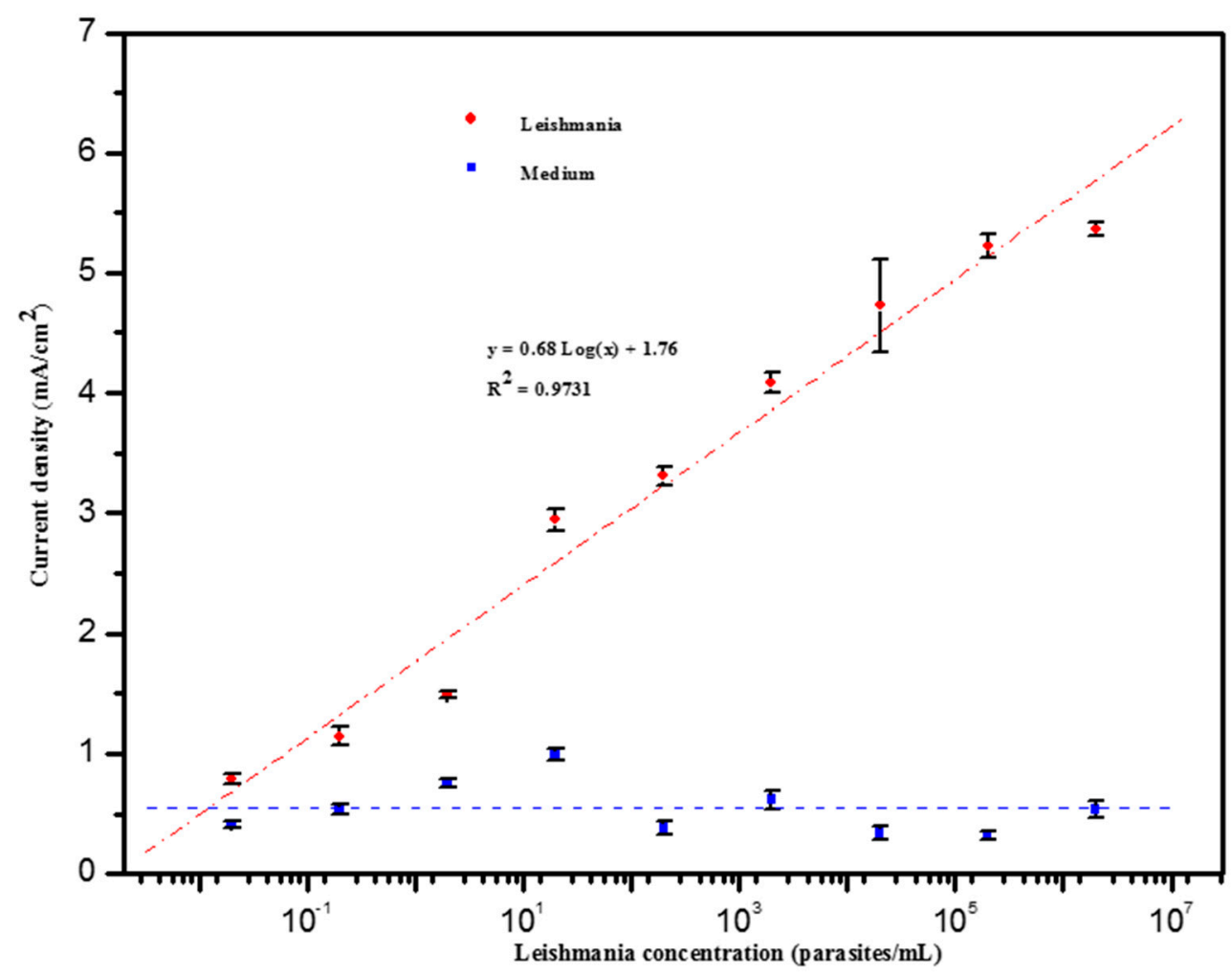

Figure 5. Biosensor response to various concentrations of Leishmania infantum parasites (logarithmic range) ranging from $2 \times 10^{-2}$ to $2 \times 10^{6}$ parasites $/ \mathrm{mL}$ and to various concentrations of parasite-free medium in $1 \mathrm{M} \mathrm{HCl}$ solution.

\subsection{Effect of the AmB Treatment}

$\mathrm{AmB}$ is considered as one of the most effective drugs for VL treatment, with a cure rate of $\sim 97 \%$ and no reported resistance $[50,51]$. Despite its widespread use, as well as its therapeutic efficiency, there is still controversy about the molecular mechanism of action of AmB. Nevertheless, the widely accepted model is based on the effects of both ergosterol binding and pore formation [52,53]. Previous study reported the inhibition of the growth of $L$. donovani in vitro by application of $\mathrm{AmB}$ at a concentration of $0.3 \mathrm{mg} / \mathrm{mL}$ [54]. Herein, the influence of incubation time of AmB with Leishmania parasites on the subsequent interaction between the latter and casein-AuNPs conjugates was investigated. A solution containing Leishmania parasites at a fixed concentration $10^{4}$ parasites $/ \mathrm{mL}$ was incubated with a $0.3 \mathrm{mg} / \mathrm{mL}$ of AmB for different time intervals, then exposed to casein@AuNPs conjugates. The effect of the incubation time on the current response, associated with the interaction of casein with Leishmania parasites, is shown on Figure 6. Hence, the current response decreased nonlinearly with the increase of the incubation time and reached a plateau after approximately $240 \mathrm{~min}$, indicating the inhibition of the casein-Leishmania interaction. An incubation time of $\sim 240 \mathrm{~min}$ seems to be appropriate for the effective inhibition of Leishmanial detection. Actually, it is not clear with which mechanism the antibiotic has made this inhibition, as revealed by chronoamperometric response decreases, e.g., either by blocking the active site of casein-GP63 interaction or simply through Leishmania lysis, which cause parasite number diminution. The experimental data are fitted with an exponential regression: (current density) $=4.2 \times \exp \left(-8 \times 10^{-2} \mathrm{t}\right)$, where the time $\mathrm{t}$ is expressed in minutes, with a correlation coefficient $\mathrm{R}^{2}=0.9569$. 

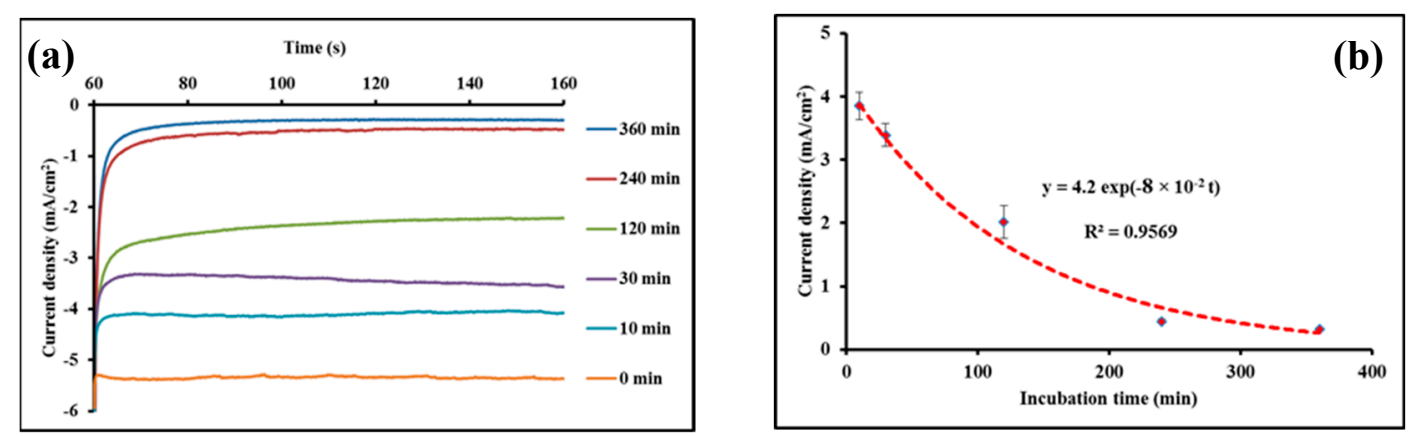

Figure 6. (a) Chronoamperometric curves obtained without AmB pretreatment of Leishmania parasites (0 min) and with AmB pretreatment for different times (10;30; 120; 240 and $360 \mathrm{~min})$. (b) corresponds to the electrocatalytical signal highlighting the effect of the incubation time of Leishmania parasites with AmB on the casein-GP63 interaction. The discontinuous line indicated the fitting of the experimental data with an exponential regression (current density) $=4.2 \times \exp \left(-8 \times 10^{-2} t\right),\left(R^{2}=0.9569, n=3\right)$.

\section{Conclusions}

In this work, AuNPs and casein@AuNPs conjugates were first synthesized, characterized by Transmission Electron Microscopy, Ultraviolet-Visible spectroscopy, and X-ray Photoelectron Spectroscopy, and used to design an electrochemical biosassay for Leishmania parasite detection and quantification by targeting its major surface protease GP63. The casein-conjugated gold nanoparticles are allowed to interact with Leishmania parasites taking advantage from the specificity of the interaction between casein and the GP63 proteins. This interaction was evaluated by chronoamperometry measurement of the current associated with AuNP catalysis of the hydrogen evolution reaction on screen-printed carbon electrodes. The assay was able to detect Leishmania with a detection limit of $\sim 0.55$ parasite $/ \mathrm{mL}$ and over a linear range from $2 \times 10^{-2}$ to $2 \times 10^{5}$ parasites $/ \mathrm{mL}$, along with good reproducibility ( $\mathrm{RSD} \approx 4 \%$ ). Briefly, it can be stated that the presented results are consistent with the development of a simple but highly accurate detection methodology of leishmania. The method could be promising for clinical application in human and veterinary medicine, especially in poor-resource settings. In this way, our future work will be devoted to the analysis of blood serums collected from both Leishmania-infected and noninfected dogs to assess the specificity and the sensitivity of the devised assay.

Author Contributions: Conceptualization, M.F.D. and O.O.; Methodology, M.F.D. and O.O. Formal Analysis, D.L.; Investigation, M.F.D., K.B., and R.I.; Writing-Original Draft Preparation, M.F.D.; Writing—Review and Editing, O.O.; Visualization, M.S.; Supervision, D.L.

Funding: This work was supported by the Tunisian Ministry for Higher Education, Research and Technology and the EU project H2020-MSCA RISE- 2014 TROPSENSE (ref. 645758).

Conflicts of Interest: The authors declare no conflicts of interest.

\section{References}

1. Alvar, J.; Cañavate, C.; Molina, R.; Moreno, J.; Nieto, J. Canine Leishmaniasis. In Advances in Parasitology; Elsevier: Amsterdam, The Netherlands, 2004; Volume 57, pp. 1-88. ISBN 978-0-12-031757-8.

2. World Health Organization. Sustaining the Drive to Overcome the Global Impact of Neglected Tropical Diseases: Second Who Report on Neglected Tropical Diseases; World Health Organization: Geneva, Switzerland, 2013; ISBN 978-92-4-156454-0.

3. Kallel, K.; Pratlong, F.; Haouas, N.; Kaouech, E.; Belhadj, S.; Anane, S.; Dedet, J.P.; Babba, H.; Chaker, E. Isoenzymatic variability of Leishmania infantum in Tunisia concerning 254 human strains. Acta Trop. 2008, 106, 132-136. [CrossRef]

4. Ndao, M. Diagnosis of Parasitic Diseases: Old and New Approaches. Interdiscip Perspect Infect Dis. 2009, 2009, 1-15. [CrossRef] [PubMed] 
5. de Paiva-Cavalcanti, M.; de Morais, R.C.S.; Pessoa-e-Silva, R.; Trajano-Silva, L.A.M.; da Cunha Gonçalves-de-Albuquerque, S.; de Hollanda Cavalcanti Tavares, D.; Brelaz-de-Castro, M.C.A.; de Freitas e Silva, R.; Pereira, V.R.A. Leishmaniases diagnosis: an update on the use of immunological and molecular tools. Cell Biosci. 2015, 5. [CrossRef]

6. Travi, B.L.; Cordeiro-da-Silva, A.; Dantas-Torres, F.; Miró, G. Canine visceral leishmaniasis: Diagnosis and management of the reservoir living among us. PLoS Negl. Trop. Dis. 2018, 12, e0006082. [CrossRef] [PubMed]

7. Oliveira, E.; Saliba, J.W.; Oliveira, D.; Dias, E.S.; Paz, G.F. A prototype of the direct agglutination test kit (DAT-Canis) for the serological diagnosis of canine visceral leishmaniasis. Vet. Parasitol. 2016, 221, 9-13. [CrossRef] [PubMed]

8. Reithinger, R.; Dujardin, J.-C.; Louzir, H.; Pirmez, C.; Alexander, B.; Brooker, S. Cutaneous leishmaniasis. Lancet Infect. Dis. 2007, 7, 581-596. [CrossRef]

9. Yao, C.; Donelson, J.E.; Wilson, M.E. The major surface protease (MSP or GP63) of Leishmania sp. Biosynthesis, regulation of expression, and function. Mol. Biochem. Parasitol. 2003, 132, 1-16. [CrossRef]

10. Olivier, M.; Atayde, V.D.; Isnard, A.; Hassani, K.; Shio, M.T. Leishmania virulence factors: focus on the metalloprotease GP63. Microbes Infect. 2012, 14, 1377-1389. [CrossRef] [PubMed]

11. Schneider, P.; Rosat, J.-P.; Bouvier, J.; Louis, J.; Bordier, C. Leishmania major: Differential regulation of the surface metalloprotease in amastigote and promastigote stages. Exp. Parasitol. 1992, 75, 196-206. [CrossRef]

12. Bouvier, J.; Schneider, P.; Etges, R.; Bordier, C. Peptide substrate specificity of the membrane-bound metalloprotease of Leishmania. Biochemistry 1990, 29, 10113-10119. [CrossRef]

13. Nogueira de Melo, A.C.; d'Avila-Levy, C.M.; Dias, F.A.; Armada, J.L.A.; Silva, H.D.; Lopes, A.H.C.S.; Santos, A.L.S.; Branquinha, M.H.; Vermelho, A.B. Peptidases and gp63-like proteins in Herpetomonas megaseliae: Possible involvement in the adhesion to the invertebrate host. Int. J. Parasitol. 2006, 36, 415-422. [CrossRef] [PubMed]

14. Liu, Y.; Guo, R. Interaction between Casein and the Oppositely Charged Surfactant. Biomacromolecules 2007, 8, 2902-2908. [CrossRef]

15. Huppertz, T.; Smiddy, M.A.; de Kruif, C.G. Biocompatible Micro-Gel Particles from Cross-Linked Casein Micelles. Biomacromolecules 2007, 8, 1300-1305. [CrossRef]

16. Liu, Y.; Guo, R. The interaction between casein micelles and gold nanoparticles. J. Colloid Interface Sci. 2009, 332, 265-269. [CrossRef]

17. Yang, T.; Li, Z.; Wang, L.; Guo, C.; Sun, Y. Synthesis, Characterization, and Self-Assembly of Protein Lysozyme Monolayer-Stabilized Gold Nanoparticles. Langmuir 2007, 23, 10533-10538. [CrossRef]

18. Jiang, X.; Jiang, J.; Jin, Y.; Wang, E.; Dong, S. Effect of Colloidal Gold Size on the Conformational Changes of Adsorbed Cytochrome $c$ : Probing by Circular Dichroism, UV-Visible, and Infrared Spectroscopy. Biomacromolecules 2005, 6, 46-53. [CrossRef] [PubMed]

19. Espinoza-Castañeda, M.; de la Escosura-Muñiz, A.; González-Ortiz, G.; Martín-Orúe, S.M.; Pérez, J.F.; Merkoçi, A. Casein modified gold nanoparticles for future theranostic applications. Biosens. Bioelectron. 2013, 40, 271-276. [CrossRef] [PubMed]

20. Jans, H.; Huo, Q. Gold nanoparticle-enabled biological and chemical detection and analysis. Chem. Soc. Rev. 2012, 41, 2849-2866. [CrossRef]

21. Singh, M.; Harris-Birtill, D.C.C.; Markar, S.R.; Hanna, G.B.; Elson, D.S. Application of gold nanoparticles for gastrointestinal cancer theranostics: A systematic review. Nanomed. Nanotechnol. Biol. Med. 2015, 11, 2083-2098. [CrossRef]

22. Cao, X.; Ye, Y.; Liu, S. Gold nanoparticle-based signal amplification for biosensing. Anal. Biochem. 2011, 417, 1-16. [CrossRef]

23. Baptista, P.; Pereira, E.; Eaton, P.; Doria, G.; Miranda, A.; Gomes, I.; Quaresma, P.; Franco, R. Gold nanoparticles for the development of clinical diagnosis methods. Anal. Bioanal.Chem. 2008, 391, 943-950. [CrossRef]

24. de la Escosura-Muñiz, A.; Maltez-da Costa, M.; Sánchez-Espinel, C.; Díaz-Freitas, B.; Fernández-Suarez, J.; González-Fernández, Á.; Merkoçi, A. Gold nanoparticle-based electrochemical magnetoimmunosensor for rapid detection of anti-hepatitis B virus antibodies in human serum. Biosens. Bioelectron. 2010, 26, 1710-1714. [CrossRef]

25. Hassan, A.-R.H.A.-A.; de la Escosura-Muñiz, A.; Merkoçi, A. Highly sensitive and rapid determination of Escherichia coli O157:H7 in minced beef and water using electrocatalytic gold nanoparticle tags. Biosens. Bioelectron. 2015, 67, 511-515. [CrossRef] 
26. de la Escosura-Muñiz, A.; Plichta, Z.; Horák, D.; Merkoçi, A. Alzheimer's disease biomarkers detection in human samples by efficient capturing through porous magnetic microspheres and labelling with electrocatalytic gold nanoparticles. Biosens. Bioelectron. 2015, 67, 162-169. [CrossRef]

27. Costa, M.M.; de la Escosura-Muñiz, A.; Merkoçi, A. Electrochemical quantification of gold nanoparticles based on their catalytic properties toward hydrogen formation: Application in magnetoimmunoassays. Electrochem. Commun. 2010, 12, 1501-1504. [CrossRef]

28. Mayorga-Martinez, C.C.; Chamorro-Garcia, A.; Merkoçi, A. Electrochemical Impedance Spectroscopy (bio)sensing through hydrogen evolution reaction induced by gold nanoparticles. Biosens. Bioelectron. 2015, 67, 53-58. [CrossRef]

29. Guerbouj, S.; Djilani, F.; Bettaieb, J.; Lambson, B.; Diouani, M.F.; Ben Salah, A.; Ben Ismail, R.; Guizani, I. Evaluation of a gp63-PCR Based Assay as a Molecular Diagnosis Tool in Canine Leishmaniasis in Tunisia. PLoS ONE 2014, 9, e105419. [CrossRef] [PubMed]

30. Sayhi, M.; Ouerghi, O.; Belgacem, K.; Arbi, M.; Tepeli, Y.; Ghram, A.; Anik, Ü.; Österlund, L.; Laouini, D.; Diouani, M.F. Electrochemical detection of influenza virus H9N2 based on both immunomagnetic extraction and gold catalysis using an immobilization-free screen printed carbon microelectrode. Biosens. Bioelectron. 2018, 107, 170-177. [CrossRef]

31. Turkevich, J.; Stevenson, P.C.; Hillier, J. A study of the nucleation and growth processes in the synthesis of colloidal gold. Spec. Discuss. Faraday Soc. 1951, 11, 55-75. [CrossRef]

32. Boisselier, E.; Astruc, D. Gold nanoparticles in nanomedicine: Preparations, imaging, diagnostics, therapies and toxicity. Chemical Society Reviews 2009, 38, 1759-1782. [CrossRef]

33. Brülle, T.; Ju, W.; Niedermayr, P.; Denisenko, A.; Paschos, O.; Schneider, O.; Stimming, U. Size-Dependent Electrocatalytic Activity of Gold Nanoparticles on HOPG and Highly Boron-Doped Diamond Surfaces. Molecules 2011, 16, 10059-10077. [CrossRef] [PubMed]

34. Kimling, J.; Maier, M.; Okenve, B.; Kotaidis, V.; Ballot, H.; Plech, A. Turkevich Method for Gold Nanoparticle Synthesis Revisited. J. Phys. Chem. B 2006, 110, 15700-15707. [CrossRef] [PubMed]

35. Liu, Y.; Xiang, Y.; Ding, D.; Guo, R. Structural effects of amphiphilic protein/gold nanoparticle hybrid based nanozyme on peroxidase-like activity and silver-mediated inhibition. RSC Adv. 2016, 6, 112435-112444. [CrossRef]

36. Casaletto, M.P.; Longo, A.; Martorana, A.; Prestianni, A.; Venezia, A.M. XPS study of supported gold catalysts: the role of $\mathrm{Au} 0$ and $\mathrm{Au}+\delta$ species as active sites. Surf. Interface Anal. 2006, 38, 215-218. [CrossRef]

37. Maye, M.M.; Lou, Y.; Zhong, C.-J. Core-Shell Gold Nanoparticle Assembly as Novel Electrocatalyst of CO Oxidation. Langmuir 2000, 16, 7520-7523. [CrossRef]

38. Haruta, M.; Daté, M. Advances in the catalysis of Au nanoparticles. Appl. Catal. A 2001, 222, 427-437. [CrossRef]

39. Alivisatos, A.P. Semiconductor Clusters, Nanocrystals, and Quantum Dots. Science 1996, 271, $933-937$. [CrossRef]

40. Sen, I.K.; Maity, K.; Islam, S.S. Green synthesis of gold nanoparticles using a glucan of an edible mushroom and study of catalytic activity. Carbohydr. Polym. 2013, 91, 518-528. [CrossRef]

41. de la Escosura-Muñiz, A.; Sánchez-Espinel, C.; Díaz-Freitas, B.; González-Fernández, Á.; Maltez-da Costa, M.; Merkoçi, A. Rapid Identification and Quantification of Tumor Cells Using an Electrocatalytic Method Based on Gold Nanoparticles. Anal. Chem. 2009, 81, 10268-10274. [CrossRef]

42. Andreadou, M.; Liandris, E.; Gazouli, M.; Mataragka, A.; Tachtsidis, I.; Goutas, N.; Vlachodimitropoulos, D.; Ikonomopoulos, J. Detection of Leishmania -specific DNA and surface antigens using a combination of functionalized magnetic beads and cadmium selenite quantum dots. J. Microbiol. Methods 2016, 123, 62-67. [CrossRef] [PubMed]

43. Rodríguez, P.; Rojas, H.; Medina, M.; Arrivillaga, J.; Francisco, Y.; Dager, F.; Piscitelli, V.; Caetano, M.; Fernández, A.; Castillo, J. Study of Functionalized Gold Nanoparticles with Anti-gp63 IgG Antibody for the Detection of Glycoprotein gp63 in Membrane Surface of Leishmania Genus Parasites. Am. J. Analyt. Chem. 2013, 04, 100-108. [CrossRef]

44. de la Escosura-Muñiz, A.; Ambrosi, A.; Merkoçi, A. Electrochemical analysis with nanoparticle-based biosystems. TrAC Trends Anal. Chem. 2008, 27, 568-584. [CrossRef] 
45. Silverman, J.M.; Clos, J.; de'Oliveira, C.C.; Shirvani, O.; Fang, Y.; Wang, C.; Foster, L.J.; Reiner, N.E. An exosome-based secretion pathway is responsible for protein export from Leishmania and communication with macrophages. J. Cell Sci. 2010, 123, 842-852. [CrossRef] [PubMed]

46. Mantel, P.-Y.; Marti, M. The role of extracellular vesicles in P lasmodium and other protozoan parasites: Extracellular vesicles in protozoan parasites. Cell. Microbiol. 2014, 16, 344-354. [CrossRef]

47. Marcilla, A.; Martin-Jaular, L.; Trelis, M.; de Menezes-Neto, A.; Osuna, A.; Bernal, D.; Fernandez-Becerra, C.; Almeida, I.C.; del Portillo, H.A. Extracellular vesicles in parasitic diseases. J. Extracell. Vesicles 2014, 3, 25040. [CrossRef] [PubMed]

48. Britton, C.; Winter, A.D.; Marks, N.D.; Gu, H.; McNeilly, T.N.; Gillan, V.; Devaney, E. Application of small RNA technology for improved control of parasitic helminths. Vet. Parasitol. 2015, 212, 47-53. [CrossRef]

49. Wang, J.; Sun, X.; Zhao, J.; Yang, Y.; Cai, X.; Xu, J.; Cao, P. Exosomes: A Novel Strategy for Treatment and Prevention of Diseases. Front. Pharmacol. 2017, 8. [CrossRef]

50. Wasan, K.M.; Wasan, E.K.; Gershkovich, P.; Zhu, X.; Tidwell, R.R.; Werbovetz, K.A.; Clement, J.G.; Thornton, S.J. Highly Effective Oral Amphotericin B Formulation against Murine Visceral Leishmaniasis. J. Infect. Dis. 2009, 200, 357-360. [CrossRef]

51. Sundar, S.; Singh, A. Recent developments and future prospects in the treatment of visceral leishmaniasis. Ther. Adv. Infect. Dis. 2016, 3, 98-109. [CrossRef]

52. Kamiński, D.M. Recent progress in the study of the interactions of amphotericin B with cholesterol and ergosterol in lipid environments. Eur. Biophys. J. 2014, 43, 453-467. [CrossRef]

53. Paila, Y.D.; Saha, B.; Chattopadhyay, A. Amphotericin B inhibits entry of Leishmania donovani into primary macrophages. Biochem. Biophys. Res. Commun. 2010, 399, 429-433. [CrossRef] [PubMed]

54. Vermeersch, M.; da Luz, R.I.; Tote, K.; Timmermans, J.-P.; Cos, P.; Maes, L. In Vitro Susceptibilities of Leishmania donovani Promastigote and Amastigote Stages to Antileishmanial Reference Drugs: Practical Relevance of Stage-Specific Differences. Antimicrob. Agents Chemother. 2009, 53, 3855-3859. [CrossRef] [PubMed]

(C) 2019 by the authors. Licensee MDPI, Basel, Switzerland. This article is an open access article distributed under the terms and conditions of the Creative Commons Attribution (CC BY) license (http://creativecommons.org/licenses/by/4.0/). 